\title{
Comparison of Semi-Automated and Manual Measurements of Left Ventricular Volume from 3D Echocardiographic Studies of Patients with Cardiomyopathy
}

\author{
J Wild ${ }^{1}$, AJ Sims², SM Fleming ${ }^{3}$, A Kenny ${ }^{3}$, A Murray ${ }^{2}$ \\ ${ }^{1}$ University of Newcastle upon Tyne, Newcastle upon Tyne, UK \\ ${ }^{2}$ Regional Medical Physics Department, Freeman Hospital, Newcastle upon Tyne, UK \\ ${ }^{3}$ Department of Echocardiography, Freeman Hospital, Newcastle upon Tyne, UK
}

\begin{abstract}
Manual measurement of left ventricular volume from $3 D$ echocardiographs is time consuming, and there is a clinical need for automatic methods. We describe a semiautomatic method which uses a 3D image gradient operator and a short-axis boundary detector. Six patients with cardiomyopathy were studied using $3 D$ echocardiography and the images were analysed to measure the repeatability of measurement by the semi-automated method, and used to compare measurements of stroke volume and ejection fraction with manual measurements. Within-patient standard deviations of the semi-automated method were $14.6 \%$ and $16.4 \%$ of the group mean end diastolic and end systolic volumes respectively, and were significantly less than the between-patient standard deviations. No differences between the semi-automated method and the manual method were found for the measurements of ejection fractions or of stroke volumes.
\end{abstract}

\section{Introduction}

Trans-thoracic echocardiography is requested most commonly for assessment of left ventricular function [1]. Two dimensional imaging can be used for quantitation of left ventricular volume, but current clinical methods [2] involve image acquisition in standard scanning planes, and calculation of volume by techniques which make assumptions about cavity shape. Three-dimensional imaging has the potential to overcome these limitations [3], but practical difficulties, such as prolonged scanning time and additional processing time for 3D image reconstruction and interpolation, have delayed its introduction into routine clinical use. Recent technological developments such as phased array (matrix) transducers have led to real-time three dimensional echocardiography (RT3DE) [4] whence 2D planar images can be extracted and displayed in real time, or near real-time, during acquisition procedures. Quantitation of 3D images is done off-line, is usually time consum- ing, and may use a sub-set of the available data. There is a clinical need for automatic methods of measurement which make full use of the spatial resolution achieved by 3D imaging.

In this paper, we describe a method for the calculation of left ventricular volume which requires minimal manual intervention, and compare its results with manual measurement for patients with cardiomyopathy.

\section{Methods}

\subsection{Patients and images}

Six patients with cardiomyopathy who were undergoing assessment for cardiac transplantation were studied using 3D trans-thoracic echocardiography. A Philips Sonos 7500 ultrasound system, with an X4 transducer (Philips Medical Systems, Andover, Massachusetts, USA) was used to obtain three dimensional echocardiographic volumes. The X4 transducer incorporates a 3000 element phased array and has a frequency range of 1.6 to $4 \mathrm{MHz}$. The ultrasound system was used in wide angled acquisition mode and the $40^{\circ} \times 45^{\circ}$ pyramidal, apical, field of view included the complete left ventricle.

All image data sets were acquired in a cardiac catheterization laboratory with patients in the left lateral decubitus position. Each data set (study) was synchronized with the $\mathrm{R}$ wave, and images were acquired during four cardiac cycles with respiration suspended. Several (median of five) studies were recorded sequentially ( $<3$ minutes) for each patient. The image data sets from each study were stored to disk, then exported for further off-line analysis.

\subsection{Manual measurement}

Off-line manual measurement of end diastolic volumes (EDV) and end systolic volumes (ESV) was made by a cardiologist (SF) from the 3D ultrasound image data sets [5]. A commercial system (TomTec Imaging Systems, Munich, Germany) was used to assist border identification, but the 
final choice of endocardial position was left to the human observer. Left ventricular volumes were estimated using an 8-plane apical rotation technique [4].

\subsection{Semi-automated measurement}

Prior to saving images for further analysis, the ultrasound system translated sampled voxel locations from polar to cartesian coordinates and interpolated unsampled voxels. One further pre-processing step - conversion to cubic voxels - was required prior to semi-automated analysis.

From each study, 3D images for end diastole and end systole were selected for further analysis. A $7 \times 7 \times 7$ image gradient kernel (edge detector), based on a 2D truncated pyramid operator [6] and extended to 3D, was applied to all selected images to generate 3D gradient magnitude images.

The ventricular apex, the centre of the mitral valve and the maximum diameter of the LV were identified manually (JW) for each study - the only manual step in the process and a series of LV short axis 2D gradient magnitude images were extracted from planes perpendicular to the LV long axis from the apex to the level of the distal end of the mitral valve leaflet.

Endocardial borders were fitted to each short axis gradient magnitude image by fitting circles [7]. Circles with a diameter greater than the upper limit of LV size for that study, or whose position and radius differed from their immediate neighbour by more than a pre-defined value were rejected, and replaced with circles linearly interpolated from their nearest neighbours. The left ventricular volume was measured by the disc summation method [4], using each of the short axis slices. The thickness of each disc was one pixel.

\subsection{Repeatability}

The semi-automated method was applied to the first two sequential studies for each patient. For each study, the end diastolic and end systolic left ventricular volumes were calculated. Analysis of variance with six patients, each with two measurements, was used to calculate the withinsubject and between-subject standard deviations of LVV, separately for end diastole and end systole. Within-subject standard deviation was a measure of the repeatability of the semi-automated method.

\subsection{Comparison}

For each patient, the mean end diastolic volume and mean end systolic volume were calculated from analysis of the first two sequential studies using the semi-automated method. From these mean values, the stroke volume and the ejection fraction were calculated. The method of Bland and Altman [8] was used to compare stroke volume and ejection fraction with the same parameters measured, from the ultrasound scans, by a human observer. The observer chose, for each patient, one of the sequential studies for analysis [5] and identified frames for end systole and end diastole.

\section{Results}

Figure 1 shows LV short axis views for one patient at end diastole at three positions along the long axis. The best fit to the endocardial border is shown by the white circles which have been calculated by the semi-automated method. Figure 2 shows LV long axis images for one patient at end diastole and end systole. The white pixels show the location of the endocardial border, as determined by the semi-automated image analysis method. Each white pixel lies at the intersection of the circle fitted to each short axis LV slice and the long axis image.

By the semi-automated method, the mean (standard deviation) end diastolic volume for the six patients was 177 (79) $\mathrm{ml}$, with a range of $89 \mathrm{ml}$ to $300 \mathrm{ml}$. The mean (standard deviation) end systolic volume for the group was 146 (74) $\mathrm{ml}$, with a range of 59 to $250 \mathrm{ml}$. The mean (standard deviation) stroke volume was 32 (13) $\mathrm{ml}$, with a range of $17 \mathrm{ml}$ to $50 \mathrm{ml}$. The mean (standard deviation) ejection fraction was $20.1 \%(9.3 \%)$ with a range of $7.2 \%$ to $34.8 \%$.

By the manual method [5], the mean (standard deviation) stroke volume was 51 (15) $\mathrm{ml}$, with a range of $37 \mathrm{ml}$ to $73 \mathrm{ml}$. The mean (standard deviation) ejection fraction was $24.3 \%(12.9 \%$ ), with a range of $11.5 \%$ to $48.0 \%$.

Table 1 shows the within and between patient components of standard deviation of EDV and ESV measured by the semi-automated method for two scans per patient. For both quantities, the between subject standard deviation significantly exceeded that of the within subject standard deviation (95\% confidence level). Within patient repeatabilities were $14.6 \%$ and $16.4 \%$ of the group mean end diastolic and end systolic volumes respectively.

Figure 3 shows the results of a comparison of the semiautomated method with the manual method for ejection fraction as a Bland-Altman plot. The bias and standard deviation of differences are given in table 2, with results of a comparison of stroke volumes.

\section{Discussion and conclusions}

We have developed a method for measuring left ventricular volume from 3D echocardiography studies. The method relies on manual intervention to identify the LV long axis, but otherwise is automatic. From images of six patients with cardiomyopathy, we have measured the repeatability of the semi-automated method, and have com- 

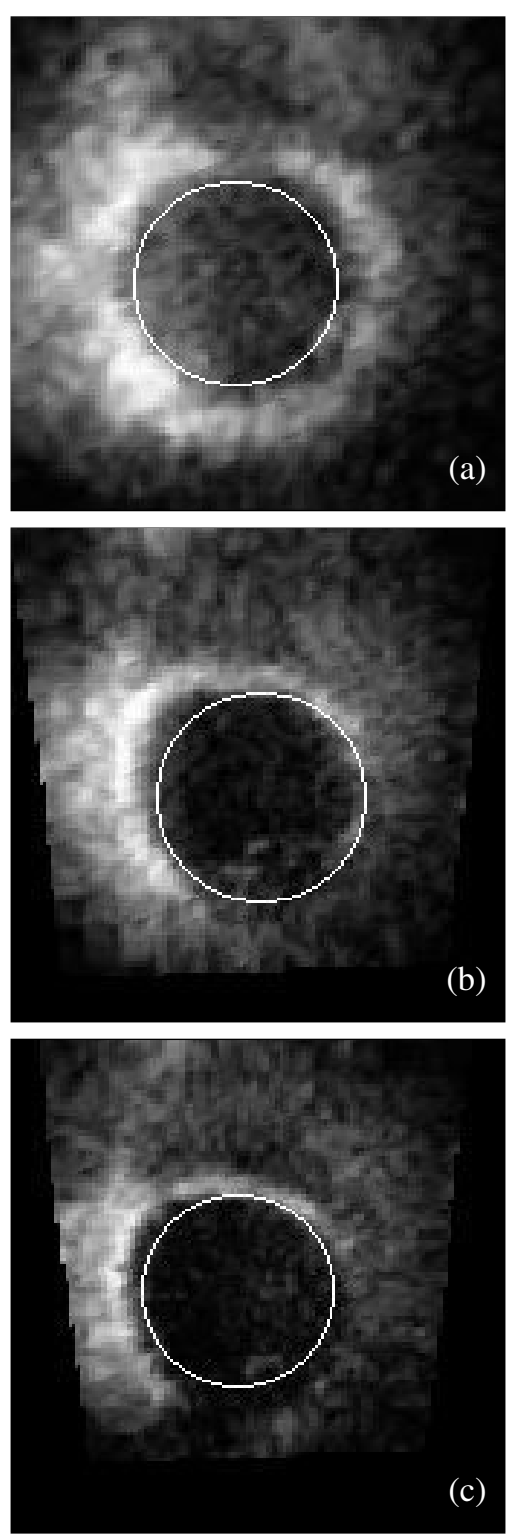

Figure 1. Short axis images of the left ventricle from patient 4 at end diastole. The planes were extracted from a 3D image, and show circles fitted to the endocardial border following application of a 3D gradient kernel and border detection. Panel (a) is at the LV base, panel (c) is near the LV apex, and panel (b) is intermediate.

Table 1. Within $\left(\sigma_{w}\right)$ and between $\left(\sigma_{b}\right)$ subject standard deviations of end diastolic left ventricular volume (EDV) and end systolic volume (ESV) measured from 3D ultrasound images by a semi-automated method. Six subjects were each scanned twice within 3 minutes.

\begin{tabular}{lccr}
\hline Volume & $\sigma_{w}(\mathrm{ml})$ & $\sigma_{b}(\mathrm{ml})$ & $P$ \\
\hline EDV & 25.9 & 76.6 & $<0.001$ \\
ESV & 23.9 & 71.9 & $<0.001$ \\
\hline
\end{tabular}
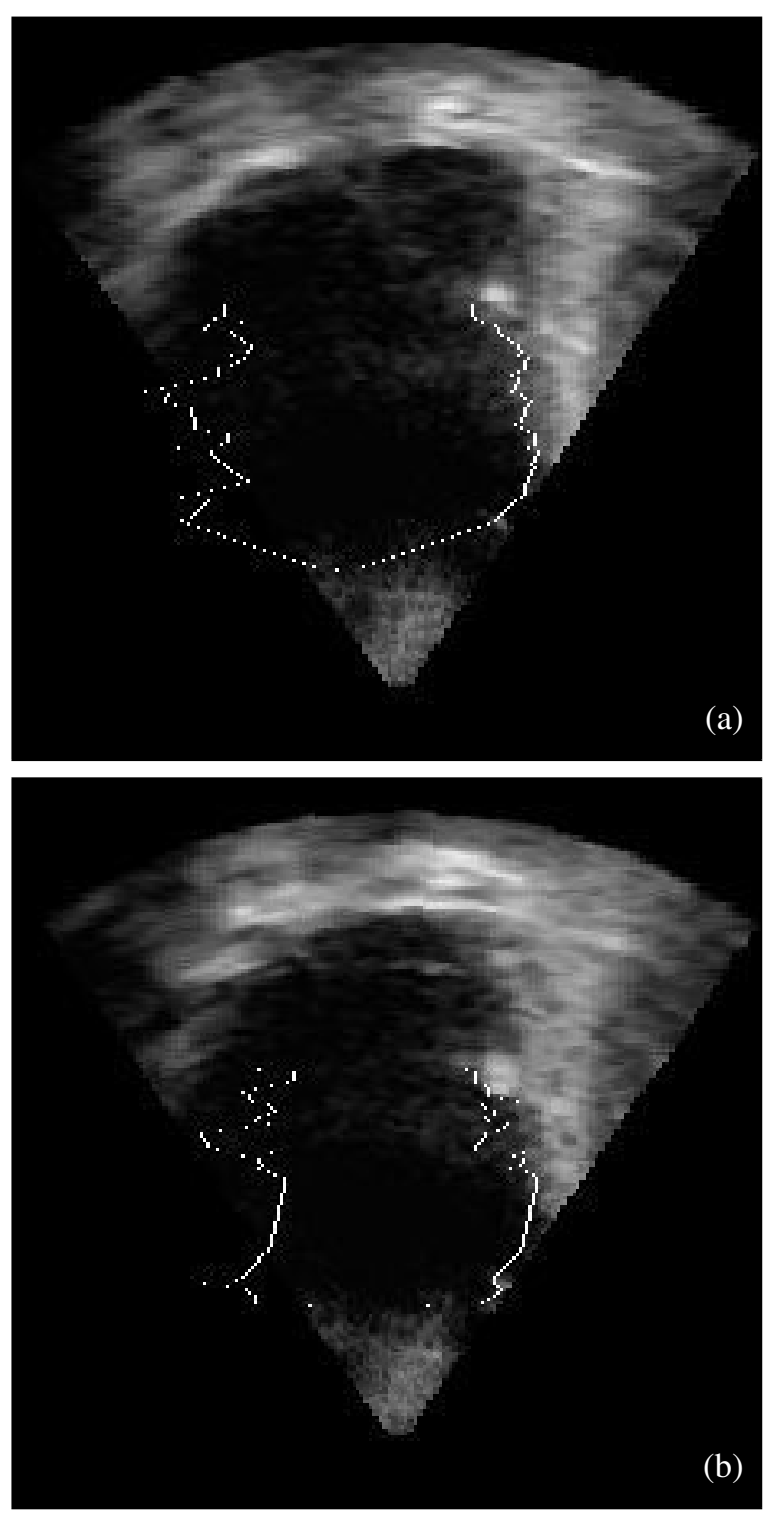

Figure 2. Long axis views of the left ventricle for patient 1 at end diastole (a) and end systole (b). White pixels show the endocardial border detected by the semiautomated method.

Table 2. Comparison of stroke volume (SV) and ejection fraction (EF), with $95 \%$ confidence intervals shown in brackets, measured by a semi-automated technique and by a manual 8-plane apical rotation method. Measurements from six subjects were compared using the method of Bland and Altman.

\begin{tabular}{lccr}
\hline Parameter & bias & std dev & $P$ \\
\hline SV $(\mathrm{ml})$ & $-19(-43$ to 4$)$ & $23(4$ to 41$)$ & 0.09 \\
EF $(\%)$ & $-4(-21$ to 12$)$ & $16(3$ to 29$)$ & 0.54 \\
\hline
\end{tabular}




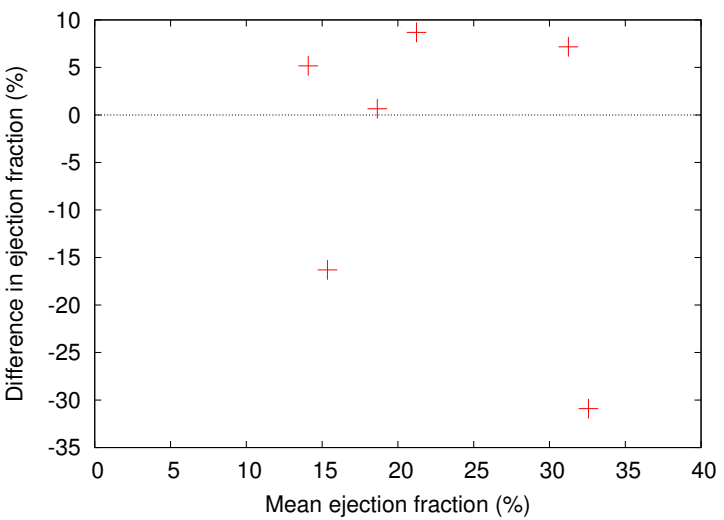

Figure 3. Comparison of ejection fraction (semiautomated method minus manual method) versus the mean of the two measurements. Table 2 gives values for the mean difference and standard deviation of differences.

pared its results with manual measurement of the same images.

For repeatability, the between patients standard deviation exceeded (at 95\% confidence level) the within patients standard deviation for measurement of end systolic and end diastolic volumes which is a necessary condition for a candidate technique for LVV measurement. Repeatabilities (14.6\% and $16.4 \%$ of the group mean end diastolic volume and end systolic volume respectively) were measured for sequential scans, and included contributions from the operator, the ultrasound system, and the boundary fitting algorithm.

Measurements of stroke volume and ejection fraction were used to compare the semi-automated method with manual measurement. No significant differences were detected between the two methods for the measurement of ejection fraction, or of stroke volumes.

These results confirm that the semi-automated method is potentially useful for measurement of chamber volume and has several merits. Notably, the three dimensional image gradient operator is able to detect surfaces, and is less sensitive to noise than its 2D counterpart. The method has several limitations. It models LV short axis endocardial cross-sections by circles, which are a poor approximation for some patient groups (including cardiomyopathy) and results in cross-sectional areas which are small compared with manual tracing. Active contour methods [9] are expected to give more accurate results.

\section{References}

[1] Hillis GS, Bloomfield P. Basic transthoracic echocardiography. BMJ 2005;330:1432-1436.

[2] Schiller NB, Shah PM, Crawford M, De Maria A, Devereaux R, Feigenbaum H, Gutgesell H, Reichek N, Sahn D, Schnittger I, Silverman NH, Tajik AJ. Recommendations for quantitation of the left ventricle by two-dimensional echocardiography. J Am Soc Echocardiography 1989;2:358-367.

[3] Siu SM, Levine RA, Rivera JM, Xie SW, Lethor JP, Handschumacher MD, Weyman AE, Picard MH. Threedimensional echocardiography improves noninvasive assessment of left ventricular volume and performance. Am Heart J 1995;130:812-822.

[4] Sugeng L, Weinert L, Lang RM. Left ventricular assessment using real-time three dimensional echocardiography. Heart 2003;89(Suppl III):iii29-iii36.

[5] Fleming SM, Cumberledge B, Kiesewetter C, Parry G, Kenny A. Usefulness of real-time three-dimensional echocardiography for reliable measurement of cardiac output in patients with ischaemic or idiopathic dilated cardiomyopathy. Am J Cardiol 2005;95:308-310.

[6] Pratt W. Digital Image Processing. Second edition. New York, USA: John Wiley \& Sons Inc, 1991.

[7] Wild J, Sims AJ, Pemberton J, Irvine T, Kenny A, Murray A. Calculation of left ventricular volume from 3D echocardiography: a comparison of three image analysis techniques. Computers in Cardiology 2004;31:61-64.

[8] Bland JM, Altman D. Statistical methods for assessing agreement between two methods of clinical measurement. Lancet 1986;1 (8476):307-310.

[9] Williams D, Shah M. A fast algorithm for active contours and curvature estimation. Comput Vision Graph 1992;55:14-26.

Address for correspondence:

Dr Andrew J Sims

Regional Medical Physics Department,

Freeman Hospital,

Newcastle upon Tyne,

NE7 7DN

United Kingdom

E-mail address: a.j.sims@newcastle.ac.uk 